\title{
OA05-02. Analysis of DNA compared to Ad5 vaccination, as single and mixed modalities, demonstrates robust induction of cellular immune responses in macaques
}

\author{
LA Hirao*1, L Wu ${ }^{1}$, A Satishchandran 1 , AS Khan², A Finnefrock ${ }^{3}$, \\ D Casimiro ${ }^{3}$, J Shiver ${ }^{3}$, MR Betts ${ }^{1}$, NY Sardesai ${ }^{2}$ and DB Weiner ${ }^{1}$
}

\author{
Address: ${ }^{1}$ Pathology, University of Pennsylvania, Philadelphia, PA, USA, ${ }^{2}$ VGX Pharmaceuticals, The Woodlands, USA and ${ }^{3}$ Merck Research \\ Laboratories, Westpoint, PA, USA \\ * Corresponding author
}

from AIDS Vaccine 2009

Paris, France. 19-22 October 2009

Published: 22 October 2009

Retrovirology 2009, 6(Suppl 3):O32 doi:10.1 I86/1742-4690-6-S3-O32

This abstract is available from: http://www.retrovirology.com/content/6/S3/O32

(c) 2009 Hirao et al; licensee BioMed Central Ltd.

\section{Background}

We have previously reported dramatic increases in immune responses induced by DNA vaccines delivered by electroporation (EP), resulting in improved control of viral replication following a SIVmac251 challenge. We compared the immunogenicity of DNA+EP to the Ad5 vaccine, the most potent recombinant viral vector for the generation of CTL responses in macaques and humans. Futhermore, we were interested in examining the effect of prime/boost strategies using these two platforms on the magnitude and phenotype of the immune response.

\section{Methods}

Three groups of rhesus macaques ( $n=5$ ) were immunized with consensus SIVmac gag, env, pol constructs with plasmid IL-12 by EP (DNA+EP), Ad5SIVgag, pol, nef (Ad5) or saline alone (Naive). The DNA+EP group received 4 immunizations and the Ad5 group received 3 immunizations. Five months following the last immunization the DNA+EP group was boosted twice with the Ad5 vaccine and vice versa. Cellular responses were assessed by IFN $\gamma$ ELISpot, CFSE proliferation, and ICS for polyfunctional populations.

\section{Results}

The Ad5 group had an early three-fold enhancement of IFN $\gamma$ responses compared to the DNA+EP group (1925 \pm 610 and $666 \pm 297$ SFU/106 PBMCs, respectively). Subse- quent Ad5 immunizations failed to boost responses while the DNA+EP group reached a robust $9776 \pm 1589$ SFU/ 106 PBMCs. Proliferation was five-fold better and the magnitude of the polyfunctional CD8+ T cell response was a log higher in the DNA+EP group compared to the Ad5 group ( $1.31 \%$ and $0.11 \%$, respectively). When boosted with DNA, the Ad5 polyfunctional response increased six-fold $(0.67 \%)$ and the DNA+EP group had a 7 -fold increase (8.28\%) following an Ad5 booster immunization.

\section{Conclusion}

Following the initial series of immunizations the DNA+EP group surpassed the Ad5 group in terms of magnitude, proliferation, and polyfunctionality. However both groups demonstrated a boost in all responses following crossover immunizations. These results have significant implications for HIV vaccine development and warrant further study. 